\title{
Abemaciclib/Letrozole Regimen
}

National Cancer Institute

\section{Source}

National Cancer Institute. Abemaciclib/Letrozole Regimen. NCI Thesaurus. Code C156377.

A chemotherapy regimen consisting of abemaciclib and letrozole that may be used as a treatment for hormone receptor (HR)-positive advanced or metastatic breast cancer. 